\title{
A System for Series Magnetic Measurements of the LHC Main Quadrupoles
}

\author{
N. Smirnov, L. Bottura, F. Chiusano, O. Dunkel, P. Legrand, S. Schloss, P. Schnizer, and P. Sievers
}

\begin{abstract}
More than 400 twin aperture lattice quadrupoles are needed for the Large Hadron Collider (LHC) which is under construction at CERN. The main quadrupole is assembled with correction magnets in a common cryostat called the Short Straight Section (SSS). We plan to measure all SSSs in cold conditions with an unprecedented accuracy: integrated gradient of the field within $150 \mathrm{ppm}$, harmonics in a range of 1 to $5 \mathrm{ppm}$, magnetic axis of all elements within $0.1 \mathrm{~mm}$ and their field direction within $0.2 \mathrm{mrad}$. In this paper we describe the automatic measurement system that we have designed, built, and calibrated. Based on the results obtained on the two first prototypes of the SSSs (SSS3 and SSS4) we show that this system meets all above requirements.
\end{abstract}

Index Terms-LHC quadrupole, magnetic axis, magnetic measurements.

\section{INTRODUCTION}

$\mathbf{L}$ HC short straight Section (SSS) [1] contains a lattice quadrupole (MQ), a dipole (MCB) and sextopole (MS) corrector in a combined MSCB package, and a second corrector package whose details depend on the location of the SSS in the LHC lattice. In Fig. 1 we show a schematic view of the arrangement of these magnets in an assembled SSS containing an octopole corrector (MO).

The magnets in a SSS, and especially the lattice quadrupole, must satisfy strict tolerances on field strength, direction, alignment and homogeneity originating from the performance objectives of the LHC. The required precision cannot be achieved without assistance and verification based on direct magnetic field and magnetic alignment measurement techniques. Considering in addition the effect of cool-down on the mechanical and magnetic alignment and the large field contributions originating from the diamagnetic properties of the superconducting cables, it is clear that cold magnetic field and axis measurements of SSSs will be vital to guarantee that specifications are met.

As a consequence, we presently plan to cold test at CERN the entire SSS production. As a necessary step to achieve this objective we have designed and produced the measurement equipment that we describe in this paper. The degree of automation and integration of the measurement system has been designed bearing in mind constraints both due to accuracy and efficiency. In this respect one speciality of our system is that it can perform simultaneous measurement of magnetic field strength, direction, higher order harmonics and axis with respect to the magnet fiducials, thus quickly providing integral and consistent information about the magnetic properties of relevance.

Manuscript received September 24, 2001.

The authors are with CERN, LHC Division, CH-1211 Geneva 23, Switzerland (e-mail: Nikolay.smirnov@cern.ch).

Publisher Item Identifier S 1051-8223(02)04266-5.

\section{SySTEM SPECIFICATIONS}

In accordance with the requirements for accelerator performance, we have set accuracy targets to be achieved by the measurement system depending on the type of magnet measured. The requirements are stricter for lattice quadrupoles than for corrector packages. The corresponding measurement accuracy targets have been expressed in Table I for the lattice quadrupoles and in Table II for the corrector packages in terms of maximum systematic and random errors. For the field measurements the error has been expressed in normalized units, i.e., $10^{-4}$ of the main field, at the LHC reference radius of $17 \mathrm{~mm}$.

\section{MEASUREMENT SYSTEM DESCRIPTION}

The SSS measurement system is a modification of an existing automatic scanner used previously for the field measurement of the first 10-m long LHC dipole prototypes [2]. The measurement principle of the original system is to use a slender rotating coil placed in the magnet bore to map the angular dependence of the field in the magnet cross-section and thus obtain information on field harmonics and field center within the frame of the rotating coil axis. A substantial modification has been undertaken on the original system in order to make it suitable for simultaneous optical and magnetic measurement of the SSSs, necessary to allow external fiducialization of the coil rotation axis.

A schematic view of the scanner is shown in Fig. 2, while Fig. 3 shows the details of the new measurement probe. The SSS is mounted on the cold test bench providing the cryogenic, powering and instrumentation interface (not shown in Fig. 2). The SSS bore is equipped with a temperature controlled warm finger (anticryostat) that provides the room-temperature support for the measurement probe. The core of the system is the probe itself, consisting of two rotating coils supported against the anti-cryostat by ceramic ball-bearings sitting in bronze cages and fitted with spring-loaded longitudinal rollers. This support, the same used for the long dipole shafts [3], allows continuous and regular longitudinal displacement of the probe still maintaining a well-defined mechanical support. The two coils in the probe have different design and functions. The 0.6-m long harmonic probe contains five identical pick up coils placed radially in a fiber-reinforced epoxy composite. This coil is used for the measurement of the main field strength and harmonics. The 0.1-m long axis probe is assembled from four identical pick up coils placed tangentially on a fiber-reinforced epoxy support. A Light Emitting Diode (LED) is accurately positioned in the center of this support, on its rotation axis, and can be surveyed from one end of the test bench. This short coil is used as described later to measure the position of the local center of the main field. 




Fig. 1. Layout of a lattice quadrupole and correctors in a LHC short straight section containing an octupole corrector package.

The rotation is transmitted to the coils from a computer-controlled rotating unit placed outside the magnet (stepping motor and gearbox, angular encoder) via a torsionally stiff, 13-m long ceramic shaft. The angular encoder in the rotating unit provides the angular reference frame for the rotation of the measurement coils. For this reason the intermediate ceramic pipe is designed to have a maximum torsion of $0.5 \mathrm{mrad}$ under normal operating conditions. Short titanium bellows are placed between the ceramic modules to accommodate geometry tolerances of the anticryostat, still maintaining torsional stiffness. For a similar reason a double-cardan joint is placed between the two coils. This avoids any lateral force acting on the delicate axis coil due to anticryostat deformations. The mechanical and electrical interface between ceramic shaft and coils in the probe is easily demountable. This feature has proven to be extremely useful for quick repair of any element, or to interchange coils for special measurements, e.g., in warm conditions.

The rotating unit can be displaced longitudinally on rails by a motorized, computer controlled transport system. The system has a reproducibility of $0.3 \mathrm{~mm}$ in longitudinal direction. As the rotating unit is moved longitudinally, a level meter mechanically fixed with respect to the angular encoder records the orientation of the rotating unit with respect to gravity.

The signal read-out during rotation of the axis coil and analyzed in Fourier series provides the location of the magnetic center of the quadrupole or higher multipole fields with respect to the coil rotation axis. To refer this to the SSS fiducials it is hence necessary to survey the location of the coil axis during the measurement. This is done in a first step by an alignment telescope equipped with a CCD camera and an on-board processor for image treatment mounted at one side of the test bench, outside the SSS. The telescope measures the position of the light spot generated by a diaphragm mounted in front of the LED. This measurement gives the $x, y$ coordinates of the coil rotation axis in a vertical plane whose $z$ position is given by the known
TABLE I

ACCURACY TARGETS FOR THE MEASUREMENT OF LATTICE QUADRUPOLES

\begin{tabular}{lccc}
\hline Measured parameters & & Absolute & Random \\
\hline Integrated gradient, transfer function & [units] & 5 & 1.5 \\
Field direction & [mrad] & 0.3 & 0.1 \\
Magnetic center & [mm] & 0.15 & 0.15 \\
Harmonics & [units] & 0.05 & 0.01 \\
\hline
\end{tabular}

TABLE II

ACCURACY TARGETS FOR THE MEASUREMENT OF CORRECTOR PACKAGES

\begin{tabular}{lccc}
\hline Measured parameters & & Absolute & Random \\
\hline Integrated field, transfer function & [units] & 10 & 5 \\
Field direction & {$[\mathrm{mrad}]$} & $0.4(\mathrm{MS})$ & 0.2 \\
& & $2(\mathrm{MO})$ & \\
Magnetic center & {$[\mathrm{mm}]$} & 0.15 & 0.15 \\
Harmonics & {$[\mathrm{units}]$} & 10 & 10 \\
\hline
\end{tabular}

location of the longitudinal positioning system. The $x, y$ coordinates in the vertical plane are given in the reference frame of the alignment telescope. Combining the information from magnetic measurement (magnetic axis with respect to coil rotation axis) and this first survey (coil rotation axis with respect to the line of sight of the alignment telescope) the magnetic axis is thus referred to the bore-sight of the alignment telescope at any desired longitudinal location.

The bore-sight is established prior to scanning the SSS using two reference positions, located upstream and downstream of the SSS in test. The reference locations are the magnetic axis of two short reference quadrupoles, whose fiducialization is well-known, stable and periodically checked. This intermediate step is necessary to materialize the bore sight by the fiducial locations on the reference quadrupoles. The final step necessary is then to measure the relative position of the reference quadrupoles and SSS fiducials. This is done using a LTD-500 laser tracker that operates in 3-D space around the test bench. 


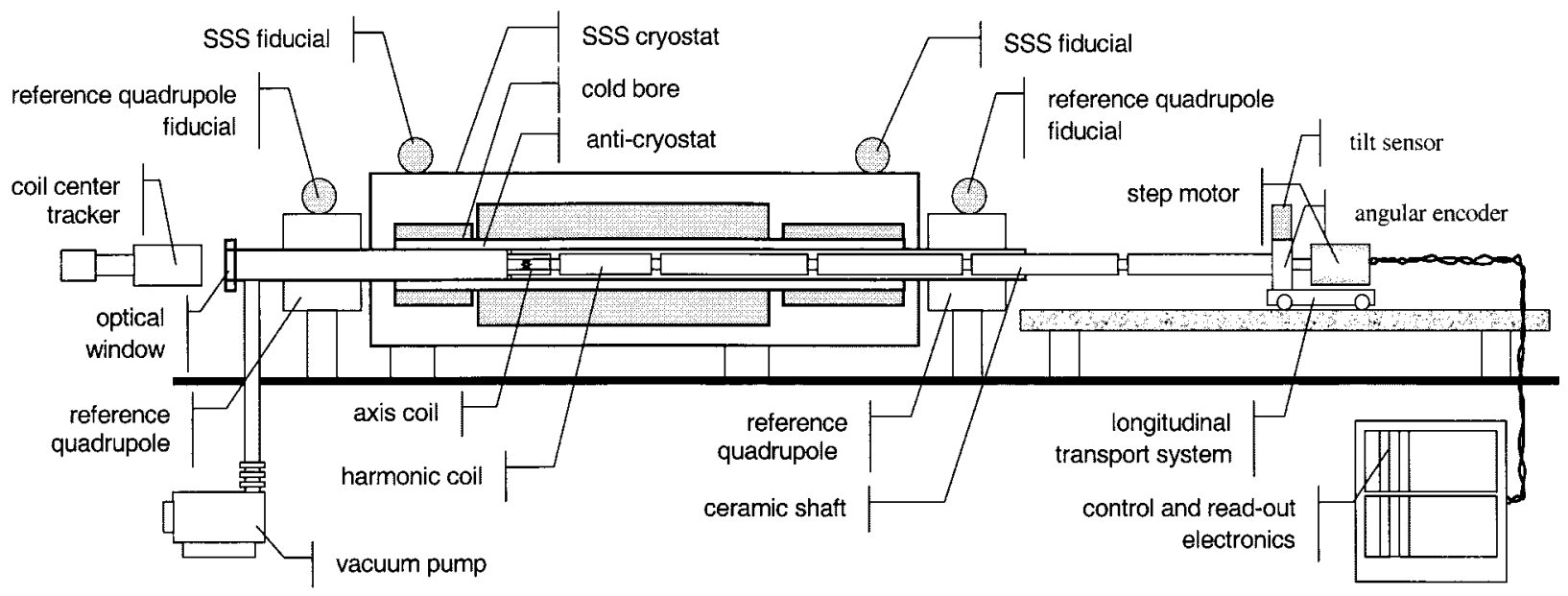

Fig. 2. Schematic view of the automated scanner system for harmonics and axis measurement on SSSs. The anti-cryostat region between the axis coil and the optical window in front of the coil tracker is evacuated during operation.

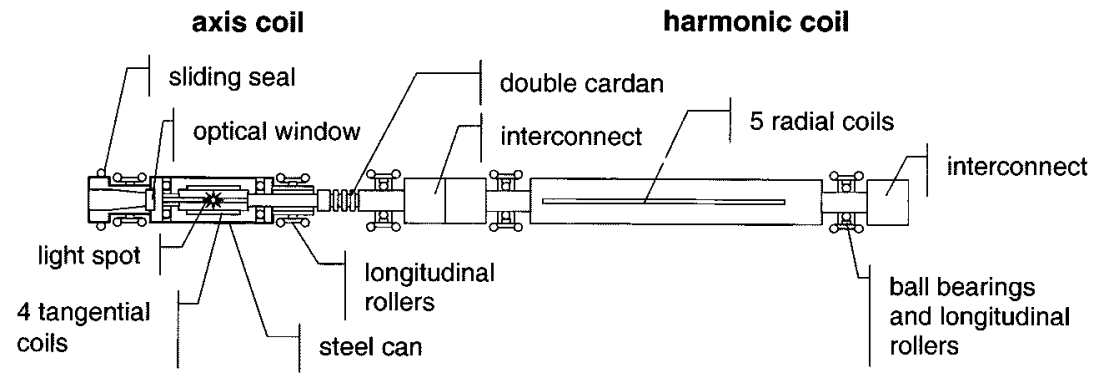

Fig. 3. Schematic view of the SSS measurement probe consisting of an assembly of a 60-cm long harmonic coil and a 10-cm long axis coil.

This rather complex procedure is further made difficult by the physical effect of light deflection in the presence of density gradients in the media traversed. In the initial feasibility studies of the system we have found that vertical temperature gradients in the anticryostat at operating conditions can be significant, reaching approximately $100 \mathrm{~K} / \mathrm{m}$. The mechanism establishing and sustaining this gradient is longitudinal convection from hot to cold regions in the anticryostat. The longitudinal gradient is transformed into a vertical gradient by the return flow in the convection cell. The temperature gradient results in a steady state density gradient (denser gas at the bottom of the anticryostat) that in turn acts as a prism on light rays. The result is that an object, such as the light spot in the axis coil, surveyed through the anticryostat appears to be several $\mathrm{mm}$ higher than in reality.

In order to avoid this effect altogether we have decided to evacuate the region between the axis coil and telescope. This is made possible by enclosing the axis coil in a steel can with a leak tight optical window at the front and a seal sliding against the anticryostat wall, and putting a leak tight optical window at the end of the anticryostat facing the telescope. The required vacuum level is moderate, approximately $1 \mathrm{mbar}$, so that little leaks during motion and cleanliness of the materials are not an issue. Proper choice of the material thickness and support avoids that the deformation of the window under the pressure differential affects the optical performance of the system. Isolation of the window from vibration sources (e.g., vacuum pump or cryogenics in the test installation) is also a critical point.

\section{QUALIFICATION OF THE MEASUREMENT System}

We have verified the performance of the system during the tests conducted on the SSS3 and SSS4 prototypes. Here we concentrate mainly on the qualification of the system with respect to the field measurement of the lattice quadrupole and the axis of all elements in the SSS. More detailed analysis of the system performance is given in [4]. For the discussion we use the normal and skew harmonic coefficients $b_{n}$ and $a_{n}$ of the complex series expansion of a 2-D magnetic field:

$$
\mathbf{B}_{y}+i \mathbf{B}_{x}=B_{r e f} \sum_{n=1}^{\infty} \frac{\left(b_{n}+i a_{n}\right)}{10^{4}}\left(\frac{x+i y}{R_{r e f}}\right)^{n-1} .
$$

In the above expansion the first term, with $n=1$, corresponds to the dipole component, and the reference field $B_{\text {ref }}$ is the main field of the magnet being analyzed. The harmonics $b_{n}$ and $a_{n}$ are expressed in units at the LHC reference radius $R_{\text {ref }}$ of $17 \mathrm{~mm}$.

\section{A. Main Field}

The harmonic coil could not be completely geometrically calibrated before the tests. We therefore have used construction data for the calculation of the coil sensitivity to the harmonics. This is particularly critical for the measurement of the main field. It was however possible to verify the accuracy of the calibration by performing five separate measurements of the quadrupole main field with the five radial coils. Using the results of this measurement we estimate that the outermost coil radius is accurate within $15 \mu \mathrm{m}$ with respect to the construction data. 


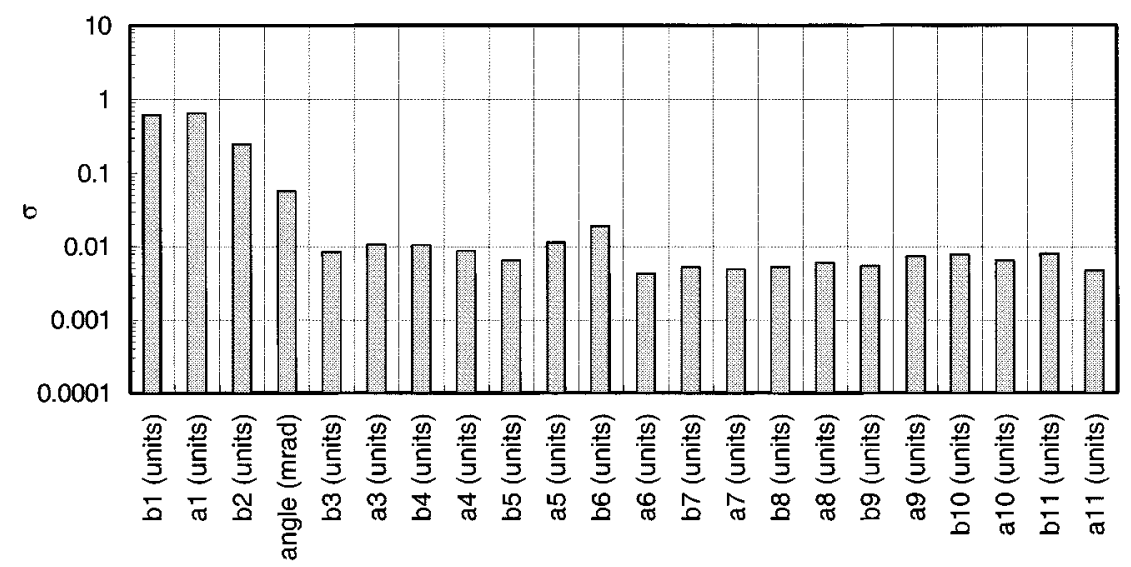

Fig. 4. Standard deviation of the field harmonics derived from measurements performed in the SSS3 prototype at $5 \mathrm{kA}$ current.

This value is coherent with construction tolerances, and corresponds to a systematic error of approximately $0.1 \%$ on the main quadrupole field, i.e., a factor two above the target accuracy. We plan to perform magnetic calibration of the radii of each coil to eliminate this systematic error to a large extent.

The resolution of the system is much higher, with a random error safely smaller than 1.5 units as shown in Fig. 4. We have established the resolution taking the standard deviation of a long sequence of measurements at a constant current $(5 \mathrm{kA}$ for Fig. 4). To achieve this result we needed to amplify the coil signal close to the source. A summary of the effect of the operation current on the random error is given in Table III.

For comparison we have reported there the resolution achieved with and without amplifier stage before the data acquisition. The effect of the amplifier is marginal at intermediate and high field, where the signal is already strong and the measurement noise is dominated by mechanics (see next section).

\section{B. Field Direction Measurement}

The field direction was measured in both apertures of the SSS prototypes at the nominal field. An absolute angle calibration was not available at the time of the measurement, and no statement can be made on the systematic error so far. However, the resolution of the system, as shown in Fig. 4 is less than $0.1 \mathrm{mrad}$ and safely within tolerance. We expect a systematic error smaller than $0.3 \mathrm{mrad}$.

\section{Field Harmonics}

The field harmonics are measured with the harmonic coil. As for the main field we have established the random error from a long sequence of measurements at a constant current of $5 \mathrm{kA}$, where the quadrupole field is sufficiently stable. The result of this analysis is shown in Fig. 4, where one can see that tolerances are safely met for all harmonics starting from the sextupole.

To analyze the origin of the random error we split the noise in two categories of electric and mechanical origin. Electric noise introduced by the coil, cables and electronics has constant amplitude and results in a random spread $\sigma_{\text {electric }}$ inversely proportional to the current. Analyzing data taken at different currents we estimate that the electric noise has average amplitude of less
TABLE III

RANDOM ERROR ON THE MAIN QUADRUPOLE FIELD

\begin{tabular}{ccc}
\hline Current (kA) & \multicolumn{2}{c}{$\sigma$ (units @ 17 mm) } \\
& with amplifier & without amplifier \\
\hline 0.73 & 1.5 & 3.0 \\
5 & 0.27 & 0.3 \\
10 & 0.17 & 0.2 \\
\hline
\end{tabular}

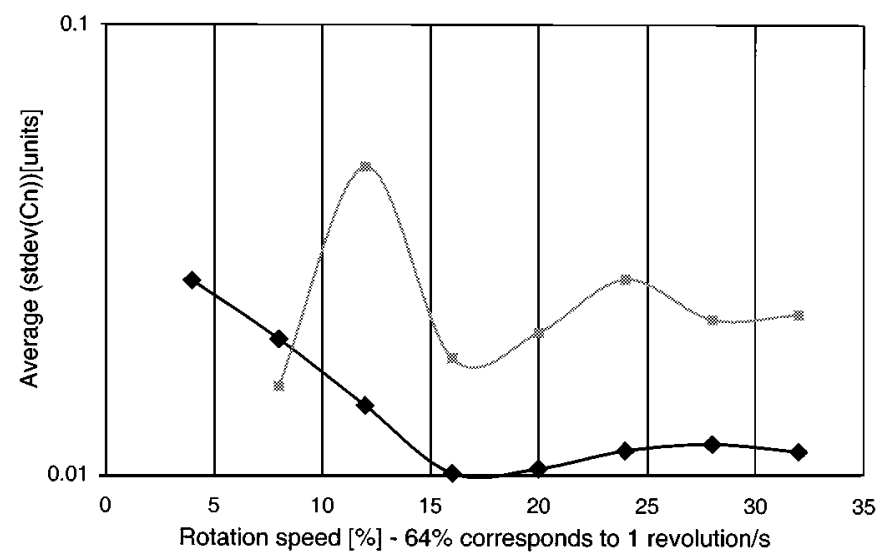

Fig. 5. Random error measured as a function of rotating speed, showing the damping effect of the axis coil on torsional vibration of the harmonic coil (diamond—with axis coil, square-without axis coil attached).

than $0.5 \mu \mathrm{V}$ [5]. The signal amplifier close to the coil is instrumental to suppress electrical noise. Without amplifier this figure increases to $1.1 \mu \mathrm{V}$ on average.

Mechanical noise is constant in relative terms (i.e., grows with increasing current) and results in a random spread $\sigma_{\text {mechanic }}$ that adds to the overall random error. Also we expect this component to depend on rotation speed and mechanical details. The order of magnitude of the random error introduced by mechanical noise after subtraction of electric noise is well below 0.01 unit for all higher harmonics and 0.05 unit for the main field.

The effect of rotation speed on the random error is shown in Fig. 5, which presents the total random error of the harmonics as measured with and without axis coil. Clear resonances can be seen without axis coil. This shows that the rotation speed has to be chosen carefully, depending on the mechanical layout, to 
avoid excitation of vibration modes in the system. The results obtained are consistent with results of computer simulation of the measurement system error performed for reasonable amplitudes of vibrations [4].

\section{Magnetic Axis Measurement}

Magnetic axis reproducibility is generally limited to outside fiducials, the errors introduced by survey procedures and axis transfer. The reason is that the magnetic center can be determined with respect to the coil rotation axis with a very high resolution, generally well below $1.0 \mu \mathrm{m}$. The standard deviation of the axis measurement is approximately $20 \mu \mathrm{m}$ in the reference frame of the alignment telescope and $60 \mu \mathrm{m}$ in the SSS fiducial frame. This value is safely within the target tolerance.

\section{CONCLUSIONS}

The automated measurement equipment developed for the magnetic field and axis measurement of the LHC SSSs satisfies most accuracy targets initially set. The system is unique in that it can measure simultaneously all magnetic properties relevant for a gradient magnet. We will continue tests and especially magnetic calibration and cross checks against other systems such as a stretched wire, to achieve full qualification in the near future. In the meantime a second unit is in preparation to increase the test capacity.

\section{REFERENCES}

[1] T. Tortschanoff et al., "The short straight sections for the LHC," in Proc. PAC-1997, pp. 3374-3376.

[2] J. Billan, J. Buckley, R. Saban, P. Sievers, and L. Walckiers, "Design and test of the benches for the magnetic measurement of the LHC dipoles," IEEE Trans. Magn., vol. 30, no. 4, pp. 2658-2661, July 1994.

[3] J. Billan et al., "Twin rotating coils for cold magnetic measurements of $15 \mathrm{~m}$ long LHC dipoles," IEEE Trans. Appl. Superconduct., vol. 10, no. 1, pp. 1422-1426, 2000.

[4] P. Schnizer, L. Bottura, M. Buzio, and N. Smirnov, “'Truffaldino,' A tool to simulate rotating coil magnetometers for accelerator magnets,", submitted for publication.

[5] L. Walckiers et al., "Sensitivity and accuracy of the systems for the magnetic measurements of the LHC magnets at CERN," in Proc. EPAC 2000, pp. 2184-2187. 